Marquette University

e-Publications@Marquette

Chemistry Faculty Research and Publications

Chemistry, Department of

$2-1-2005$

Hydroxy double salt anion exchange kinetics: effects of precursor structure and anion size

Everson Kandare

Marquette University

Jeanne Hossenlopp

Marquette University, jeanne.hossenlopp@marquette.edu

Accepted version. Journal of Physical Chemistry B, Vol. 109, No. 17 (February 2005): 8469-8475.

DOI. (C) 2005 American Chemical Society. Used with permission. 
NOT THE PUBLISHED VERSION; this is the author's final, peer-reviewed manuscript. The published version may be accessed by following the link in the citation at the bottom of the page.

\title{
Hydroxy Double Salt Anion Exchange Kinetics: Effects of Precursor Structure and Anion Size ${ }^{\dagger}$
}

\author{
Everson Kandare \\ Department of Chemistry, Marquette University, \\ Milwaukee, WI \\ Jeanne M. Hossenlopp \\ Department of Chemistry, Marquette University, \\ Milwaukee, WI
}

\begin{abstract}
H}$ NMR spectroscopy and powder X-ray diffraction have been used to explore the details of anion exchange reactions of two layered hydroxy double salts (HDSs), zinc copper hydroxy acetate (ZCA), nickel zinc hydroxy acetate (NZA), and a related layered material, zinc hydroxy acetate (ZHA), at room temperature $\left(21-22^{\circ} \mathrm{C}\right)$. Reactions that followed Avrami-Erofe'ev kinetics with respect to temporal profiles for acetate release, ZCA with butyrate $\left(k=1.7 \times 10^{-3} \mathrm{~s}^{-1}\right)$, and octanoate $\left(k=0.79 \times 10^{-3} \mathrm{~s}^{-1}\right)$ anions, as well as ZHA with octanoate $\left(k=2.6 \times 10^{-3} \mathrm{~s}^{-1}\right)$, demonstrate that rate constants for acetate release are influenced by the exchange anion relative size as well as by the solid precursor structure/composition. The reaction of NZA with octanoate deviated from expected Avrami-Erofe'ev behavior, with

Journal of Physical Chemistry B, Vol 109, No. 17 (February 2005): pg. 8469-8475. DOI. This article is (C) American Chemical Society and permission has been granted for this version to appear in e-Publications@Marquette. American Chemical Society does not grant permission for this article to be further copied/distributed or hosted elsewhere without the express permission from American Chemical Society.
\end{abstract}


evidence for an intermediate species in the solid phase that may influence the rate of acetate release into solution. The reaction of ZCA with formate anions exhibited a unique zeroth-order kinetics release of acetate, providing the possibility of developing tunable nanostructured anion release sources by use of variations in the size of the exchange species.

\section{Introduction}

A variety of layered inorganic/organic hybrid compounds with nanodimensional interlayer spacings that contain exchangeable anions have been developed for applications such as catalysis, water purification, ion exchange, fire retardancy, and controlled release of anions. In particular, layered double hydroxides (LDHs) and basic metal hydroxides have been the subject of numerous studies. 1 LDHs, with a generic formula of $\left[\mathrm{M}^{2+}{ }_{1-x} \mathrm{M}^{3+}{ }_{x}(\mathrm{OH})_{2}\right]^{x+} \mathrm{A}_{x}{ }^{-} \cdot z \mathrm{H}_{2} \mathrm{O}$, consist of positively charged metal hydroxide layers containing divalent and trivalent metal ions and inorganic or organic anions, $A^{-}$, that serve to balance the charge as well as control the interlayer spacing. LDHs can be considered complementary to cationic, or smectite, clays, and the ability to exchange intercalated ions has been a key factor in optimizing these materials for different applications. Similarly, anion exchange reactions have been utilized with basic metal hydroxides such as intercalated copper hydroxide, $\mathrm{Cu}_{2}(\mathrm{OH})_{3} \mathrm{X}$, where $\mathrm{X}$ is an intercalated anion. The magnetic properties of intercalated copper hydroxides have been shown to exhibit a striking dependence on the interlayer spacing. $\underline{2-4}$

Hydroxy double salts, HDSs, are another more recently emerging class of compounds that hold similar promise for development as new nanostructured materials for a wide range of applications. HDSs, $\left[\left(\mathrm{M}^{2+}{ }_{1-x}, \mathrm{Me}^{2+}{ }_{1+x}\right)(\mathrm{OH})_{3(1-y)}\right]^{+} \mathrm{A}^{n-}(1+3 y) / n^{*} \cdot \mathrm{zH}_{2} \mathrm{O}$, are similar to LDHs except that the metal hydroxide layer contains two divalent metals, designated here as $\mathrm{M}^{2+}$ and $\mathrm{Me}^{2+}$. The identity of the metal ions controls the details of the intralayer structure. The exchangeable anion, $\mathrm{A}^{n-}$, can either be monovalent, for example, $\mathrm{Cl}^{-}$, $\mathrm{NO}_{3}{ }^{-}$, or $\mathrm{CH}_{3} \mathrm{COO}^{-}$, or divalent, such as $\mathrm{SO}_{4}{ }^{2-}$ or $\mathrm{CO}_{3}{ }^{2-}$. Different synthetic routes for obtaining HDSs have been reported, and there have been several studies that characterize anion-exchanged materials. $\underline{5-8}$ 
Although there has been some work reported on the synthesis and characterization of HDSs, no studies to date have focused on the kinetics and mechanism of the anionic exchange reactions. However, in the case of LDHs and other related lamellar materials, time-resolved energy-dispersive X-ray diffraction, (EDXRD), has been utilized to obtain in situ kinetic data on the evolution of the solid-phase structures during intercalation and/or anion exchange reactions. $\frac{9-15}{}$ Anionic exchange reactions are generally viewed as topotactic solidstate methods of modifying existing layered materials while maintaining their overall structural integrity. This is possible because of the rapid diffusion of guest anions into and out of the 2-D interlamellar spaces. Several factors including structural morphology, intrinsic and extrinsic defects, lattice vacancies, lines and planes of deformation, interstitials, and grain boundaries play an important role in the reactivity of these materials. In addition to following the evolution of the solid structure, there have been a few reports where similar exchange reactions have been examined from the perspective of determining the temporal profiles of anion release into solution. 16,17 Characterizing anion release rates is a critical aspect in the design of nanostructured materials for applications such as the controlled release of drugs.

By analogy to LDH compounds, some key factors in HDS anion exchange reactivity are expected to be reactant structural parameters (metal ion identity and intralayer structure), crystallite size and structural disorder, and the identity/structure of the replacement anion. The goal of this study is to determine the kinetics of acetate release from selected acetate-containing HDSs in order to characterize the structural factors that influence the kinetics in these reactions. Proton nuclear magnetic resonance (NMR) spectroscopy is used to determine the kinetics of acetate release following exposure of three model compounds to $n$-alkyl carboxylate anions. The corresponding solid products obtained by quenching the reaction at selected times are analyzed via powder X-ray diffraction to obtain complementary information on the structural transformation of the HDSs; characterizing the structure of quenched reaction products is critical for tailoring the fabrication of exchange products with desired physical or chemical properties for specific applications. The results obtained 
here provide the first detailed view of the kinetics and mechanisms of HDS anion exchange reactions.

\section{Experimental Section}

Copper acetate monohydrate, $(98.0 \%)\left[\mathrm{Cu}\left(\mathrm{CH}_{3} \mathrm{COO}\right)_{2} \cdot \mathrm{H}_{2} \mathrm{O}\right]$, zinc acetate, (99.9\%) [ $\left.\mathrm{Zn}\left(\mathrm{CH}_{3} \mathrm{COO}\right)_{2}\right]$, nickel acetate tetrahydrate, (98.0\%) $\left[\mathrm{Ni}\left(\mathrm{CH}_{3} \mathrm{COO}\right)_{2} \cdot 4 \mathrm{H}_{2} \mathrm{O}\right]$, and zinc oxide, $(99.9 \%)$ [ $\left.\mathrm{ZnO}\right]$ were obtained from Aldrich Chemical Co. Sodium salts of formate, butyrate, propionate, and octanoate, with at least $98.0 \%$ purity, and HPLCgrade methanol (99.9\%) were also obtained from Aldrich Chemical Co. $\mathrm{D}_{2} \mathrm{O}(99.9 \%)$ was obtained from Cambridge Isotope Laboratories, Inc. All chemicals were used without further purification.

The hydroxy double salts were prepared by following a literature coprecipitation method ${ }^{8}$ as follows: zinc copper hydroxy acetate (ZCA) was prepared by mixing $0.41 \mathrm{~g}$ of $\mathrm{ZnO}(5 \mathrm{mmol})$ with $1.00 \mathrm{~g}$ of $\mathrm{Cu}\left(\mathrm{CH}_{3} \mathrm{COO}\right)_{2} \cdot \mathrm{H}_{2} \mathrm{O}(5 \mathrm{mmol})$ in $10 \mathrm{~mL}$ of deionized water with vigorous stirring at room temperature. The resultant suspension was allowed to stand for $24 \mathrm{~h}$, after which the precipitate was filtered off and washed several times with deionized water before drying at room temperature. The nickel zinc hydroxy acetate (NZA) HDS and zinc hydroxy acetate (ZHA) were prepared in a similar way, maintaining the starting molar ratio of $\mathrm{ZnO}$ to the corresponding metal acetate as $1: 1$. In some instances, a second treatment with nickel or zinc acetate was necessary to ensure that there was not any remaining polycrystalline $\mathrm{ZnO}$ in the product.

The acetate-containing HDS or ZHA was mixed with $0.2 \mathrm{M}$ solutions of the target sodium salt of the exchange anion dissolved in $\mathrm{D}_{2} \mathrm{O}$. Multiple reaction mixtures were prepared by mixing $30 \mathrm{mg}$ of the original HDS powder with $3 \mathrm{~mL}$ of the exchange solution followed by mechanical shaking. After a range of time intervals, filtering the dispersion halted the reaction. The residue was dried at room temperature prior to X-ray diffraction analysis, and the filtrate was stored in sealed vials to be used for NMR analysis. Complete exchange of the acetate from ZCA was also attempted by exposing ZCA to exchange anion solutions for $48 \mathrm{~h}$, filtering, and repeating the process with a fresh exchange solution. 
Powder X-ray diffraction (PXRD) patterns were obtained using a Rikagu diffractometer operated in a para-focusing Bragg-Bretano configuration, with a $1 / 2^{\circ}$ divergence slit, $1 / 2^{\circ}$ scatter slit, $0.15-\mathrm{mm}$ receiving slit, $0.15-\mathrm{mm}$ monochromator receiving slit, a $\mathrm{Cu} \mathrm{Ka}(\lambda=$ $0.154 \AA$ ) radiation source operated at $1 \mathrm{~kW}$, and data acquisition was performed using a step size of $0.036 \% / 20 \mathrm{~s}$. Samples were mounted on microscope slides using $10 \%$ GE 7031 varnish in ethanol after confirming that the varnish mixture did not perturb the observed patterns. The instrument response was obtained using the National Institute of Standards and Technology (NIST) standard reference material Si powder (SRM 640C). Peak positions and widths were determined by fitting the data to pseudo-Voigt functions using XFIT, $\underline{18}$ removing the contribution of the $\mathrm{Cu} \mathrm{Ka}_{2}$ wavelength.

The removal of acetate by exchange with other $n$-alkyl carboxylates at room temperature was followed using solution ${ }^{1} \mathrm{H}$ NMR spectroscopy on a 300-MHz Varian NMR spectrometer. No evidence for species other than acetate or the exchange anion was observed. Selected resonance intensities of nonoverlapping acetate and the exchange anion peaks were integrated for filtrates obtained at different exchange times, and data were averaged from three separate NMR spectra. Integrated intensities of the acetate methyl ${ }^{1} \mathrm{H}$ NMR peak at $1.84 \mathrm{ppm}$ were also converted to concentrations, normalizing for run-to-run variations by use of an internal standard of methanol in $\mathrm{D}_{2} \mathrm{O}$. Octanoate concentrations were determined using the integrated intensity of the triplet at $2.09 \mathrm{ppm}$ corresponding to the $\mathrm{CH}_{2}$ adjacent to the carboxyl group. Two sets of control experiments were also performed. Aliquots of the liquid portion of a reaction mixture (ZCA plus octanoate) were removed by pipet at two times, 1200 and 3600 $s$, and the acetate and octanoate concentrations were obtained via NMR analysis for comparison with data obtained via the filtration quenching scheme. A sample of ZCA was also placed in $\mathrm{D}_{2} \mathrm{O}$ with no exchange anion present and was subjected to vigorous shaking for $4500 \mathrm{~s}$, after which the liquid phase was also analyzed via NMR for production of free acetate via dissolution or other nonexchange-related degradation of the starting material.

Fourier transform infrared (FTIR) spectra of the solid materials were obtained using the $\mathrm{KBr}$ method on a Nicolet Magna-IR 560 
spectrometer operated at $1-\mathrm{cm}^{-1}$ resolution in the $400-4000-\mathrm{cm}^{-1}$ region. Diffuse reflectance spectra (DRS) of the HDS samples were taken on a Shimadzu UV-2501PC in reflectance mode at room temperature using $\mathrm{BaSO}_{4}$ as a reference, with the DRS absorbance spectra recalculated using the Kubelka-Munk equation. Elemental analysis was carried out by Huffman Labs, Colorado, using atomic emission spectroscopy interfaced with inductively coupled plasma (AES-ICP) for metals determination. ZCA:

$\mathrm{Cu}_{3.6} \mathrm{Zn}_{1.4}(\mathrm{OH})_{7.6}\left(\mathrm{CH}_{3} \mathrm{CO}_{2}\right)_{2.4} \cdot 5 \mathrm{H}_{2} \mathrm{O}$, [Cu (37.2\% calcd, 35.6\% exptl), $\mathrm{Zn}$ (14.9\% calcd, $14.8 \%$ exptl), C (9.4\% calcd, 9.4\% exptl), H (4.1\% calcd, 3.2\% exptl)]. ZHA: $\mathrm{Zn}_{5}(\mathrm{OH})_{8}\left(\mathrm{CH}_{3} \mathrm{CO}_{2}\right)_{2} \cdot 4 \mathrm{H}_{2} \mathrm{O}$, [ $\mathrm{Zn}(50.1 \%$ calcd, $48.8 \%$ exptl), C (7.4\% calcd, $7.6 \%$ exptl), $\mathrm{H}(3.4 \%$ calcd, $3.25 \%$ exptl)]. NZA: $\mathrm{Ni}_{1.5} \mathrm{Zn}_{3.2}(\mathrm{OH})_{7.9}\left(\mathrm{CH}_{3} \mathrm{CO}_{2}\right)_{1.5} \cdot 1.7 \mathrm{H}_{2} \mathrm{O}[\mathrm{Ni}(14.2 \%$ calcd, $14.9 \%$ exptl), Zn (33.7\% calcd, 35.7\% exptl), C (5.8\% calcd, $7.6 \%$ exptl), $\mathrm{H}(2.5 \%$ calcd, $3.25 \%$ exptl)]. The layered acetate compounds have a nominal exchange capacity of approximately 3 mequiv/g.

\section{Results and Discussion}

The details of the HDS structure and composition are influenced by the reaction conditions, and only limited structural information has been reported ${ }^{8}$ for compounds synthesized via the method used here. We note that our NZA samples are substantially enriched in $\mathrm{Zn}$ compared to most HDSs prepared via other synthetic routes; $;, 19$ however, HDSs with $\mathrm{Zn} / \mathrm{Ni}$ ratios of $1.8,{ }^{6}$ similar to the value of 2.1 found here, have also been observed. In addition to the elemental analysis reported in the Experimental Section, the acetate-containing reactants were characterized via PXRD, DRS, and FTIR spectroscopy.

NZA and ZHA are expected $6-8,19$ to have a modified-brucite-like HDS structure where the metal hydroxide layer is composed of octahedrally coordinated (by $\mathrm{OH}$ ) metal ions. Up to $25 \%$ of the octahedral sites are vacant, with tetrahedrally coordinated zinc ions situated directly above and below the vacancies. Anions bind to the tetrahedral sites. Figure 1A shows the PXRD data for NZA (bottom) and ZHA (top). Three equally spaced basal plane reflections, labeled $00 /(I=1$ to 3$)$, are observed; note that the PXRD data have not been indexed and hence the labeling of basal planes used here for NZA and 
ZHA does not indicate assigned Miller indices. Broad and asymmetrically shaped peaks are observed at higher angles, a result of turbostratic effects emanating from translational disorder of the metal hydroxide sheets along the $a$ and $b$ axes, which destroys the line shapes of the $(h k 0)$ reflections. $\frac{20}{}$ The interlayer $d$ spacings for these materials are similar, 13.0 for NZA and $13.6 \AA$ for $\mathrm{ZHA}$, and are comparable to the respective literature values of 12.8 and $13.2 \AA$ reported previously for these materials. ${ }^{8}$ After correction for instrument response, the observed widths of the 001 peaks were used with the Scherrer equation, $\underline{21}$ assuming a shape factor of 0.9 , to obtain estimates of the average crystallite sizes in the $c$-axis dimension: 270 $\pm 40 \AA$ for NZA and $350 \pm 45 \AA$ for ZHA.
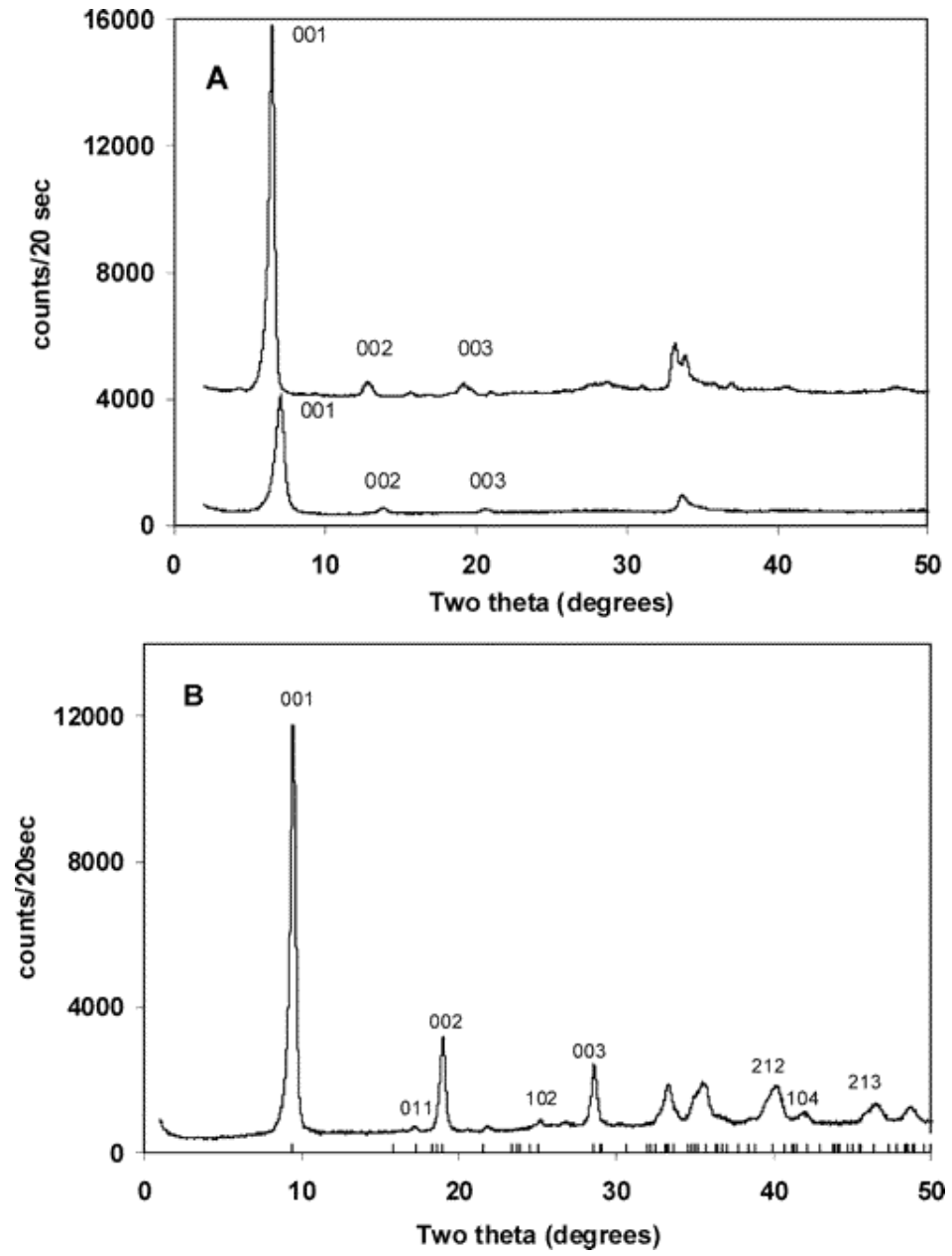

Figure 1 (A) PXRD data for NZA HDS (lower trace) and ZHA (upper trace). (B) PXRD data for ZCA HDS. Lines on the bottom indicate positions predicted for the monoclinic $P 2_{1} / m$ structure (see text for details). Miller $h k l$ indices for selected reflections are given. 
DRS and FTIR spectra of NZA (Supporting Information) are nearly identical to those reported for NZA synthesized via other routes. DRS features can be assigned to octahedrally coordinated nickel. It is difficult to obtain detailed structural information about anion orientation from $\mathrm{X}$-ray diffraction data because these materials do not form good single crystals. The dominant mode of carboxylate interaction with the metal in the tetrahedral site, $\mathrm{Zn}^{2+}$ for both NZA and $Z H A$, is typically inferred from the difference between infrared carboxylate symmetric, $\mathrm{v}_{\mathrm{s}}$, and asymmetric, $\mathrm{vas}_{\mathrm{as}}$, stretching modes, $\Delta v_{\text {as -s, }}$ as compared with known model compounds. ${ }^{22-24}$ Although there can be some ambiguity in using this method to assign the carboxylate-metal bonding, particularly ${ }^{23}$ when $\Delta v_{\text {as }-s}$ is less than 200 $\mathrm{cm}^{-1}$, the observed shift from $\Delta v_{\text {as }-\mathrm{s}}=91 \mathrm{~cm}^{-1}$ in zinc acetate (bidentate) to approximately $154 \mathrm{~cm}^{-1}$ for acetate bound to $\mathrm{Zn}^{2+}$ in our HDSs can be assigned to primarily unidentate binding ${ }^{19}$ with possible hydrogen bonding interactions of the acetate "free" oxygen to neighboring $\mathrm{OH}$ groups or to intercalated water molecules.

The structures of copper-containing HDSs are commonly compared with botallackite, $\mathrm{Cu}_{2}(\mathrm{OH})_{3} \mathrm{Cl}$, where the copper ions occupy two crystallographically distinct sites. The diffraction pattern of copper hydroxy acetate also provides a good comparison with the ZCA data shown in Figure 1B. In the copper hydroxy acetate structure, $\underline{25}$ three distinct copper sites were identified (two in a mirror plane), and the copper ions were found in Jahn-Teller distorted octahedral coordination with oxygen from the $\mathrm{OH}$ or acetate groups. The acetate group positions were found to be disordered in a 50:50 fashion via a mirror plane bisecting the $\mathrm{CH}_{3}-\mathrm{C}=\mathrm{O}$ angle. Intercalated water molecules were also observed between the layers. Although our PXRD data are not sufficient for a complete structural analysis, we note that our data are also consistent with a monoclinic $P 2_{1} / m$ structure with nearly identical lattice constants. The lines at the bottom of Figure 1B show the expected positions using CELREF unit cell assignment software, $\underline{26}$ fitting a monoclinic $P 2_{1} / m$ to obtain $a=5.60 \AA, b=6.13 \AA$, $c=9.38 \AA$, and $\beta=91.16^{\circ}$; indexing for selected peaks is also shown. Analysis of the positions of the $00 /$ peaks gives a value of $9.3 \AA$ for the $d$ spacing in ZCA, comparable to the literature value of $9.4 \AA$ for $Z C A^{8}$ and significantly smaller than the spacing observed with NZA or ZHA. 
The average crystallite size along the $c$-axis dimension was found from the 001 peak width to be $540 \pm 30 \AA$.

The FTIR spectrum of ZCA (Supporting Information) is nearly identical to that of pseudo-bridging acetate in $\mathrm{Cu}_{2}(\mathrm{OH})_{3}\left(\mathrm{CH}_{3} \mathrm{CO}_{2}\right) \cdot \mathrm{H}_{2} \mathrm{O}$ where one acetate oxygen is bound to $\mathrm{Cu}^{2+}$ and the other forms a hydrogen bond with an adjacent $\mathrm{OH} .{ }^{25}$ Elemental analysis is consistent with at least some of the acetate groups binding to $\mathrm{Cu}^{2+}$; we also note that DRS data (Supporting Information) for ZCA and selected anionexchanged HDSs are also consistent with anion interaction with $\mathrm{Cu}^{2+}$ sites based on shifts in visible wavelength bands assignable to $\mathrm{Cu}^{2+}$ $\mathrm{d}-\mathrm{d}$ transitions.

The ability to exchange anions in these materials was first tested by utilizing typical complete exchange conditions where a ZCA sample was exposed to multiple aliquots of solutions containing a different $n$-alkyl carboxylate. Figure 2 shows the variation of the product basal spacing with alkyl chain length. The interlayer spacing is dependent on chain length as well as the orientation dictated by the space-filling density of interlayer anions. From the slope of the plot of $d$ spacing versus the number of carbon atoms, the angle of orientation of the carboxylate anions can be found from the relation $\sin ^{-1}(a)=$ slope/2.54 with the assumption that the alkyl chains exhibit an alltrans conformation in the interlayer space. 27 The arrangement of the alkyl chains in the zinc copper HDS is consistent with a bilayer arrangement and a tilt angle of $52.9^{\circ}$ with respect to the hydroxylated sheets of ZCA.

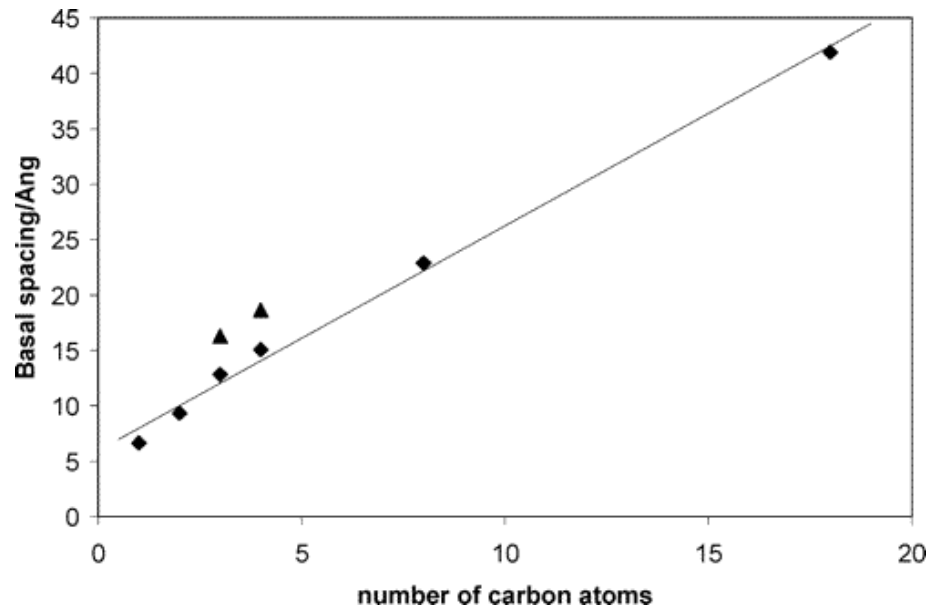

Figure 2 Interlayer spacings for Zn/Cu HDS $n$-alkyl homologous series where $n$ is the total number of carbon atoms contained in a given carboxylate. All basal spacings

Journal of Physical Chemistry B, Vol 109, No. 17 (February 2005): pg. 8469-8475. DOI. This article is (c) American Chemical Society and permission has been granted for this version to appear in e-Publications@Marquette. American Chemical Society does not grant permission for this article to be further copied/distributed or hosted elsewhere without the express permission from American Chemical Society. 
were calculated using an average of 001,002 , and 003 powder $\mathrm{X}$-ray diffraction peaks. Propionate and butyrate exhibited two sets of basal spacings.

The exchange products of propionate and butyrate each revealed the presence of two phases as evident from extra peaks in the PXRD patterns at lower diffraction angles. In all other cases, only one product phase was identified. The additional phase could be due to the coexistence of both gauche and trans arrangements of the exchange anions in the interlayer spacing or possibly the presence of two phases with different degrees of hydration hence different basal spacings, as has been reported for longer anions exchanged into copper hydroxy acetate. ${ }^{3}$ We also note that Fujita and Awaga reported that they were unable to exchange butyrate with acetate in $\left(\mathrm{Cu}_{2}(\mathrm{OH})_{3} \mathrm{X}\right)$, citing decomposition ${ }^{2}$ as a possible difficulty. Clearly, the systematic study of alkyl chain length effects in the formation of the product phases is critical for understanding the mechanism of the exchange processes, particularly when attempting to control interlayer spacing systematically.

To test the reliability of the filtration quenching method used in our kinetics studies, a control experiment was performed for the release of acetate from ZCA by exchange with octanoate. The concentrations of acetate and octanoate following reaction for 1200 and $3600 \mathrm{~s}$ were determined by NMR analysis for liquid samples obtained by the filtration quenching method as well as by directly removing an aliquot of the liquid sample. Acetate concentrations were found to be independent of sampling method for each reaction time, within the $5 \%$ uncertainty of NMR integrations. Octanoate concentrations were found at each time to be $7-8 \%$ higher in the directly sampled solutions than in the filtered solutions, suggesting that filtration sampling may lead to small systematic error in octanoate concentrations. We also note that in the absence of any exchange anion (i.e., in pure $\mathrm{D}_{2} \mathrm{O}$ ) less than $5 \%$ of the acetate content of ZCA was released when the sample was vigorously agitated for $4500 \mathrm{~s}$, demonstrating the stability of the HDS precursor in $\mathrm{D}_{2} \mathrm{O}$.

The kinetics of acetate release upon exposure to exchange anions were monitored via ${ }^{1} \mathrm{H}$ NMR analysis of filtrate solutions. NMR intensities from the original anion and the exchange anion were integrated and subsequently converted to a dimensionless quantity, 
the extent of reaction (a). In a simple topotactic exchange process, the rate of anion release would be expected to follow the same general kinetic behavior as the structural changes in the solid. On the basis of models shown to work for exchange kinetics determined from in situ analysis of LDH solid-phase structures, the data were first tested using the Avrami-Erofe'ev nucleation-growth model28-30 of a general functional form as described below.

$$
\alpha=1-\exp [-(k t)]^{m}
$$

a ranges from zero at the start of the reaction to unity, the point where the acetate concentration in solution no longer changes, and is calculated using eq 2 :

$$
\alpha=\left[\frac{I_{\text {westate }}(t)}{I_{\text {aectate }}(t)}\right]
$$

where $I_{\text {acetate }}(t)$ represents the integrated ${ }^{1} \mathrm{H}$ NMR intensity of the $\mathrm{CH}$ resonance peak of the acetate anion at time $t$ and $I_{\text {acetate }}\left(t_{\infty}\right)$ is the integrated intensity after the exchange reaction is complete. The Sharp-Hancock method ${ }^{30}$ was applied to obtain linear plots of the form

$$
\ln [-\ln (1-\alpha)]=m \ln (t)+m \ln (k)
$$

from which $m$ and $k$ values can be easily evaluated. The value of $m$ can, in certain cases, be related to the mechanism of the exchange reaction in terms of nuclei growth and or 2-D diffusion, whereas $k$ is the rate constant for the anionic exchange reaction. Several other models are available in the literature for modeling kinetic data of this nature, ${ }^{30}$ but the 2-D diffusion/nucleation model is the only one that performed satisfactorily, within the applicable a range of 0.15 to 0.85 , for most cases here.

Figure 3A shows the extent of reaction data for acetate release as well as loss of octanoate, $\mathrm{CH}_{3}\left(\mathrm{CH}_{2}\right)_{6} \mathrm{CO}_{2}^{-}$, from solution following reaction with ZCA HDS at $21.0 \pm 0.5^{\circ} \mathrm{C}$. Sharp-Hancock analysis of the acetate data, Figure 3B, gives $k=(0.79 \pm 0.17) \times 10^{-3} \mathrm{~s}^{-1}$ and $m$ $=0.79 \pm 0.03$. The value of $m$ gives an indication of the extent to 
which the exchange reaction is diffusion- or nucleation-controlled. An $m$ value of about 0.5 would suggest a wholly diffusion-controlled reaction where the progression of the reaction is solely dependent on the rate at which the exchange anion diffuses to the front of the growing intercalation phase. The value of 0.8 observed here suggests that both nucleation and 2-D diffusion dependence contribute to the overall rate of the anion release via exchange.
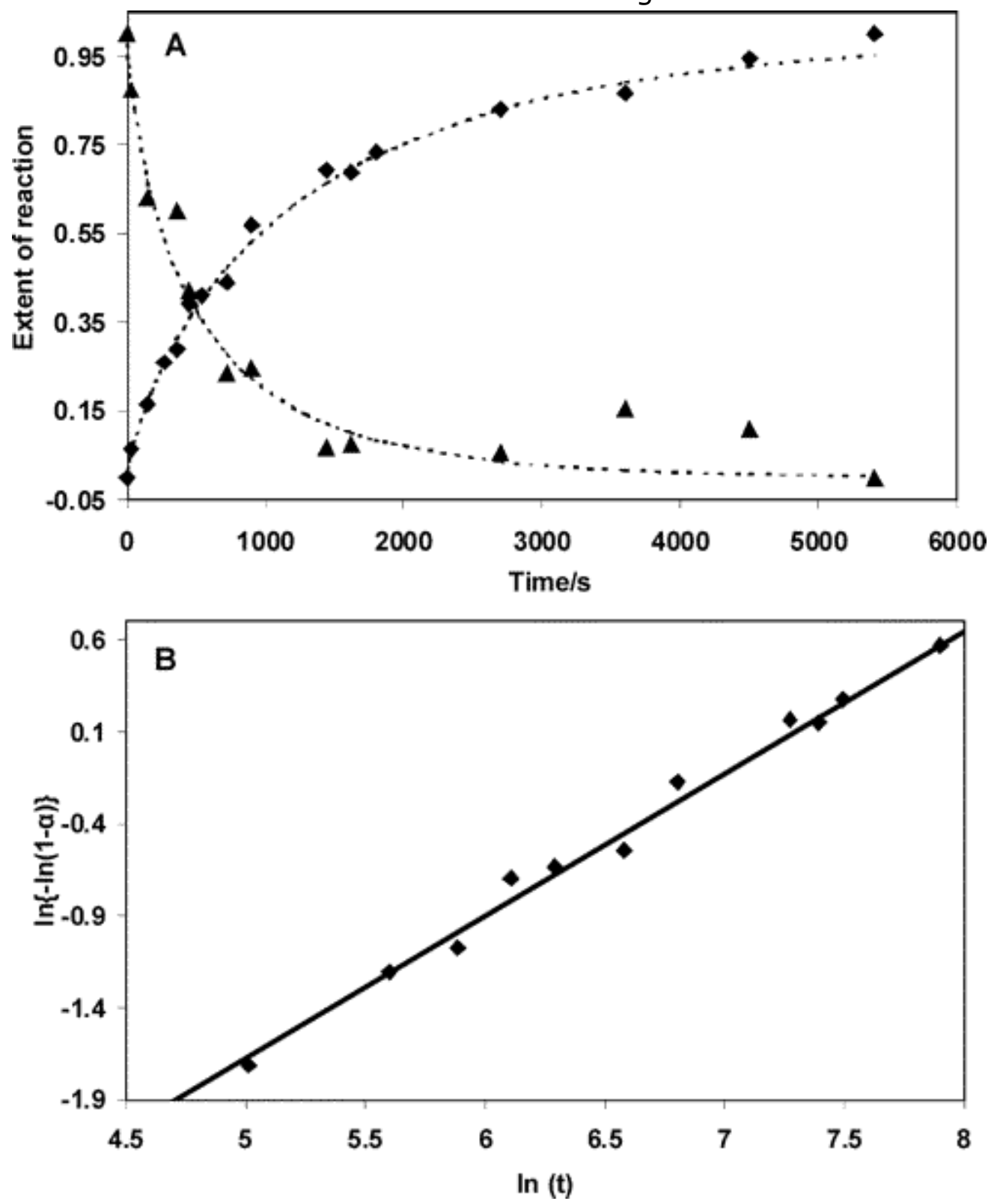

Figure $3(A)$ Extent of reaction data for the release of acetate $(\diamond)$ into solution and the corresponding loss of octanoate $(\square)$ from solution obtained for reaction with ZCA HDS. Acetate and octanoate concentrations were independently obtained from ${ }^{1} \mathrm{H} N M R$

Journal of Physical Chemistry B, Vol 109, No. 17 (February 2005): pg. 8469-8475. DOI. This article is (C) American Chemical Society and permission has been granted for this version to appear in e-Publications@Marquette. American Chemical Society does not grant permission for this article to be further copied/distributed or hosted elsewhere without the express permission from American Chemical Society. 
analysis. The fit obtained with Sharp-Hancock analysis is shown as a dashed line. (B) Corresponding Sharp-Hancock plot for acetate release data.

Comparison of acetate concentrations in solution with the original composition of the HDS enables us to determine that $98 \%$ of the acetate anions contained in the original ZCA sample had been released into solution at the conclusion of the reaction. However, higher concentrations of the octanoate were lost from solution, and the extent of reaction curves do not cross at exactly 0.5 , as would be expected for a simple topotactic exchange process. Control experiments discussed previously suggest that the filtration quenching leads to lower octanoate concentrations in the filtrate, consistent with nonspecific binding of the octanoate in the wet solid-phase sample. Elemental analysis of a completely exchanged and washed sample is, however, consistent with a one-for-one exchange of octanoate for acetate. Only the acetate concentrations, which are independent of liquid-phase sampling methods, are used here for the determination of kinetic parameters.

The effects of the exchange anion on the observed acetate release kinetics are shown in Figure 4A, where acetate concentrations are plotted as a function of time. The exchange with butyrate also shows Avrami-Erofe'ev-type behavior, and the results of Sharp-Hancock analysis are shown in Table 1. Even though the exchange efficiency was higher in the octanoate anion case, $98 \%$, as compared to $55 \%$ for butyrate, the rate constant for reaching a stable concentration of released acetate was found to be a factor of 2 larger for butyrate. The use of formate as the exchange anion led to strikingly different temporal behavior for acetate release as shown in the inset of Figure 4A. These data were not consistent with Avrami-Erofe'ev kinetics and instead followed simple zeroth-order behavior over the measured time range. To obtain the rate constant listed in Table 1, a(t) was calculated for the formate reaction by estimating the maximum possible acetate concentration based on the precursor elemental analysis and plotting $\mathrm{a}(\mathrm{t})=$ [acetate observed $]_{t} /[\text { maximum acetate }]_{\mathrm{t}}$ versus time. 
NOT THE PUBLISHED VERSION; this is the author's final, peer-reviewed manuscript. The published version may be accessed by following the link in the citation at the bottom of the page.
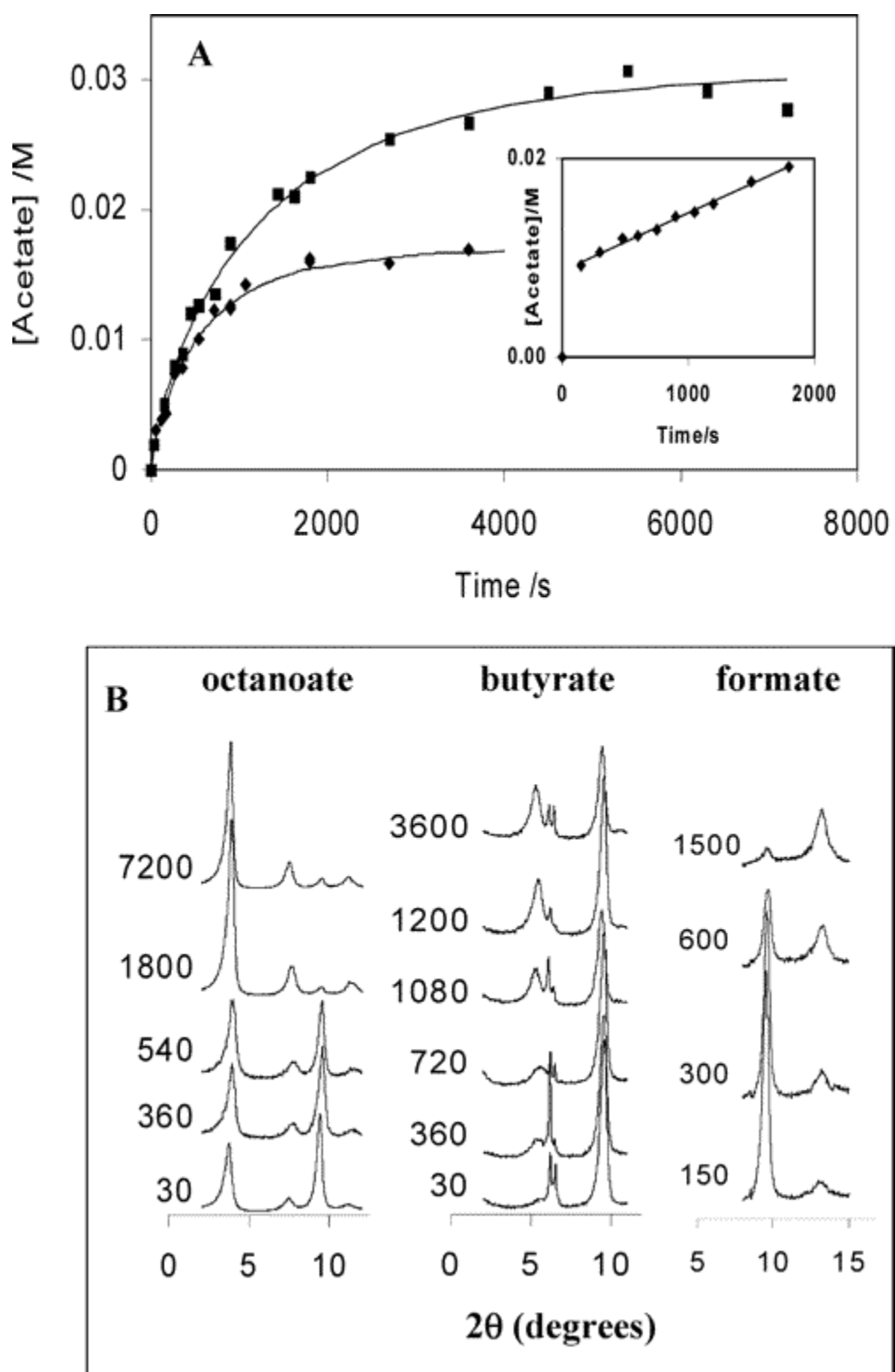

Figure $4(A)$ Acetate solution phase concentrations as a function of time following exposure of ZCA HDS to butyrate $(\bullet)$ and octanoate $(\cdot)$ anions. The inset shows acetate release following exposure of ZCA HDS to formate. (B) PXRD data for solid collected at times (seconds) indicated following exposure to each of the three anions. Data are offset for clarity but not otherwise scaled.

Table 1: Kinetic Data for Exchange Reactions

$\begin{array}{lllll}\text { exchange anion } & \mathrm{HDS} \text { formula } & \text { temp } \pm 0.5\left({ }^{\circ} \mathrm{C}\right) & m & k\left(10^{-3} \mathrm{~s}^{-1}\right) \\ \text { formate } & \text { ZCA } & 21.0 & \mathrm{~N} / \mathrm{A} & 0.19 \pm 0.06^{a} \\ \text { butyrate } & \text { ZCA } & 22.0 & 0.80 \pm 0.05 & 1.7 \pm 0.6 \\ \text { octanoate } & \text { ZCA } & 21.0 & 0.79 \pm 0.03 & 0.8 \pm 0.2 \\ \text { octanoate } & \text { ZHA } & 22.0 & 0.62 \pm 0.03 & 2.6 \pm 0.8\end{array}$

Journal of Physical Chemistry B, Vol 109, No. 17 (February 2005): pg. 8469-8475. DOI. This article is (C) American Chemical Society and permission has been granted for this version to appear in e-Publications@Marquette. American Chemical Society does not grant permission for this article to be further copied/distributed or hosted elsewhere without the express permission from American Chemical Society. 
NOT THE PUBLISHED VERSION; this is the author's final, peer-reviewed manuscript. The published version may be accessed by following the link in the citation at the bottom of the page.
octanoate
NZA
22.0
$(0.54 \pm 0.03)^{b}$
$(1.0 \pm 1.73)^{b}$

${ }^{a}$ Rate constant determined from zeroth-order kinetic fitting model. ${ }^{b}$ Kinetic parameters obtained using the Sharp-Hancock plot shown in Figure 6A do not adequately represent the full time course of the reaction.

PXRD data were obtained for the solid samples collected during the kinetics experiments in order to provide insight into the structural transformations taking place during the exchange process. Selected regions of the PXRD data for the reaction of each of the three exchange anions with ZCA are shown in Figure 4B. Reaction times increase moving from bottom to top, with the time (in seconds) indicated for each PXRD trace. In each case, the 001 peak from the ZCA HDS can be observed at $2 \theta=9.5^{\circ}$ at the earliest reaction times (found in the bottom trace of each data set). For exchange with octanoate, shown on the left side of Figure $4 B$, the only other peaks that are observed are $00 /(I=1-3)$ reflections from the zinc copper hydroxy octanoate HDS product. The structural transformation of the solid material in the formate exchange, shown on the right side of Figure 4B, follows a similar pattern of conversion of ZCA HDS to a single-phase product.

In contrast to octanoate and formate reactions, the exchange with butyrate exhibits more complex behavior in terms of solid product phases. PXRD patterns obtained from butyrate exchange, shown in the middle panel of Figure 4B, show two sharp peaks at $2 \theta$ values of 6.1 and $6.4^{\circ}$, corresponding to basal spacings of 14.5 and $13.7 \AA$, respectively, with fluctuating relative intensities. Another broader peak at $2 \theta=5.4^{\circ}$, corresponding to a $d$ spacing of $16.5 \AA$, was found to grow with increasing time of exchange. As noted previously, butyrate exchange led to products with two different $d$ spacings (15.1 and 18.6 $\AA$ ) when carried out under complete exchange conditions in $\mathrm{H}_{2} \mathrm{O}$ at room temperature. The use of $\mathrm{D}_{2} \mathrm{O}$ for NMR studies may be a factor in the small differences in basal spacings if interlamellar water is a factor because hydrogen bonding is stronger in $\mathrm{D}_{2} \mathrm{O}$ than in $\mathrm{H}_{2} \mathrm{O}$. 31 The lower overall conversion for the exchange of acetate with butyrate is also evident in the persistence of the acetate peak at $2 \theta=9.5^{\circ}$.

Anionic exchange reactions involve many competitive processes. These may include the energy needed to disrupt the hydrated structures in solution, electrostatic interactions between anions and Society and permission has been granted for this version to appear in e-Publications@Marquette. American Chemical Society does not grant permission for this article to be further copied/distributed or hosted elsewhere without the express permission from American Chemical Society. 
the cations both in solution and in the HDS, and subsequent energy gained when the exchange anion finally coordinates to the metal center in the HDS lamellar structure. $\underline{32}$ Using a simple kinetic model, such Avrami-Erofe'ev behavior, to model such multifaceted processes cannot give completely detailed mechanistic insight but does provide a basis for comparison in cases where such kinetics are followed; however, both kinetic and thermodynamic factors must be considered. Although butyrate exchange has a larger rate constant, the absolute extent of reaction is lower than that observed with octanoate exchange. Greater stability for longer alkyl chain exchange HDS products has also previously been noted in complete exchange studies. $\underline{33}$ However, there is an energetic cost associated with the larger structural change of opening the layers that must accompany exchanges involving larger anions that may serve to slow the process. Diffusion rates of the exchange anions may also play a role in determining the observed kinetics because the value of $m$ extracted from these data is consistent with both nucleation and diffusion contributions. However, despite the formation of more than one product structure in the butyrate reaction, the kinetics still follow Avrami-Erofe'ev behavior, and the larger rate constant (relative to that of the octanoate reaction) is consistent with both the diffusion and nucleation aspects of the reaction. Additional experiments, including kinetics studies of the reverse reactions and investigation of additional exchange anions, will be helpful in further elucidating the factors that control the extent of reaction as well as the number of observed product phases. We note that a preliminary study carried out in our laboratory indicates that when the butyrate exchange is carried out at $40{ }^{\circ} \mathrm{C}$ only one product phase ( $d$ spacing $=15 \AA$ ) is formed under complete exchange conditions in $\mathrm{H}_{2} \mathrm{O}$. Further work is in progress to characterize the temperature effects on these reactions.

The exchange of formate for acetate exhibits significantly different kinetic behavior compared to other ZCA HDS reactions studied here as well as those reported for LDHs or other layered compounds. The key difference is likely the fact that formate is a smaller anion. As the reaction proceeds from the edges toward the center, the interlayer spacing decreases. This pinching in of the edges may slow the diffusion of the acetate out from between the layers. This relatively uncommon behavior has been observed for certain 
solid-state dehydration reactions where desorption at the interface is the rate-limiting step. ${ }^{30}$ This result has significance for the design of these materials for controlled release applications; the relative size of the exchanging anions can be exploited to provide nanostructural changes in the solid that result in either a constant release over some time period of the target species or, for cases following Avrami-Erofe'ev kinetics, a deceleratory release.
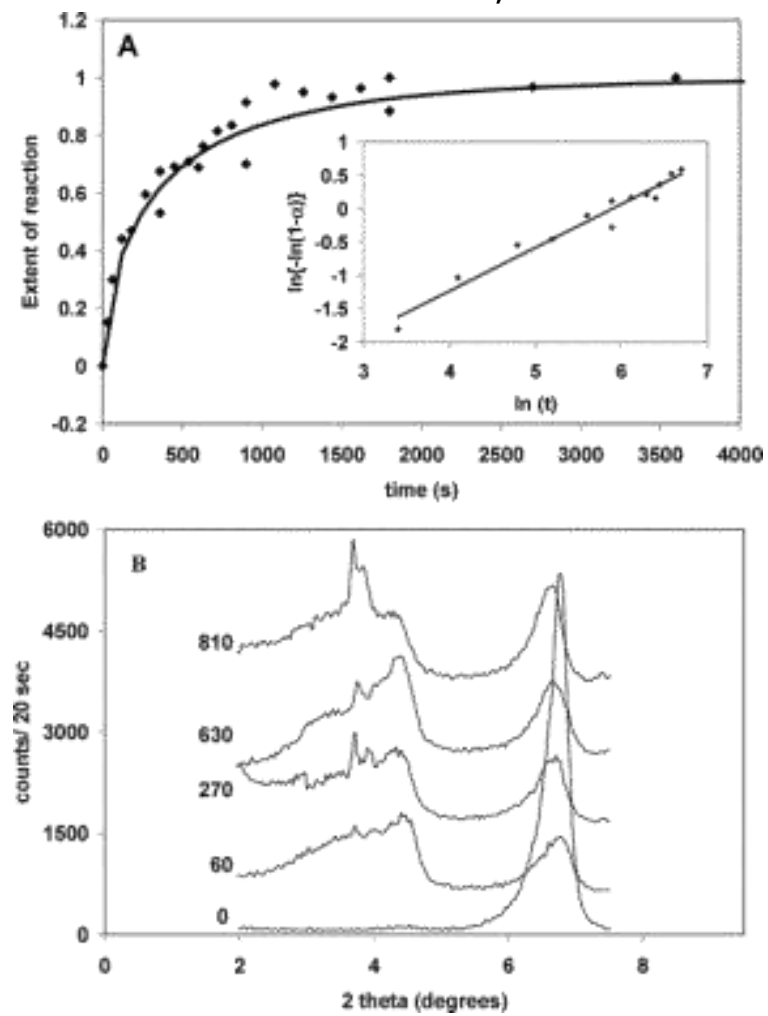

Figure 5 (A) Extent of reaction as a function of time for acetate anions released $(\bullet)$ after ZHA is exposed to octanoate solution. The inset shows the corresponding Sharp-Hancock plot for acetate release. (B) PXRD data obtained for solid materials recovered from the reaction of ZHA with octanoate. Time of exposure to exchange solutions (in seconds) is indicated for each trace. Plots are offset for clarity but not otherwise scaled.

Octanoate anions were intercalated into different host materials under the same conditions to investigate the effect of host structure and morphology on the rate of anionic exchange reactions. NZA and ZHA have similar interlayer spacings (13.0 and $13.6 \AA$, respectively, versus $9.3 \AA$ for $Z C A$ ) and $c$-axis crystallite sizes (270 and $350 \AA$, respectively, versus $540 \AA$ for $Z C A$ ), and both have $Z \mathrm{n}^{2+}$ in the tetrahedral anion binding sites. Figure 5A shows the extent of reaction as a function of time and the corresponding Sharp-Hancock plot for 
octanoate exchange into ZHA; the kinetics data are summarized in Table 1. The acetate release kinetics again follow Avrami-Erofe'ev kinetics. The reaction proceeds to completion in terms of acetate release more quickly than that of the ZCA HDS, but with a lower overall conversion. Although the acetate release data can be analyzed using the Avrami-Erofe'ev model, we note that the solid materials harvested during the reaction exhibit a greater degree of structural disorder than was observed for ZCA exchange reactions. PXRD data for the corresponding solid products are shown in Figure 5B. A very broad, asymmetric feature at $2 \theta<5^{\circ}$ is observed at short times. By $810 \mathrm{~s}$, a sharper, more intense peak consistent with the fully exchanged octanoate product is beginning to emerge from the background. The structural transformation of ZHA via exchange with octanoate is clearly more complex than that of ZCA reacting with the same anion.

Whereas NZA and ZHA have many structural similarities, the acetate release profiles upon exchange with octanoate are significantly different for the two host materials. The extent of reaction curve for NZA reacting with octanoate, shown in Figure 6A, does not follow the simple deceleratory behavior of the Avrami-Erofe'ev model. At approximately $500 \mathrm{~s}$, the acetate concentration has reached about half of its final value and appears to be leveling off, only to begin to increase again more rapidly at around 1000 s, suggesting a change in mechanism for the acetate release. We note that two separately synthesized samples of NZA HDS exhibited the initial leveling off of acetate release at $\sim 500 \mathrm{~s}$. The apparent mechanistic change is not as obvious from the Sharp-Hancock plot inset in Figure 6A, but the fit parameters clearly are not a good representation of the temporal behavior of the acetate release. 
NOT THE PUBLISHED VERSION; this is the author's final, peer-reviewed manuscript. The published version may be accessed by following the link in the citation at the bottom of the page.
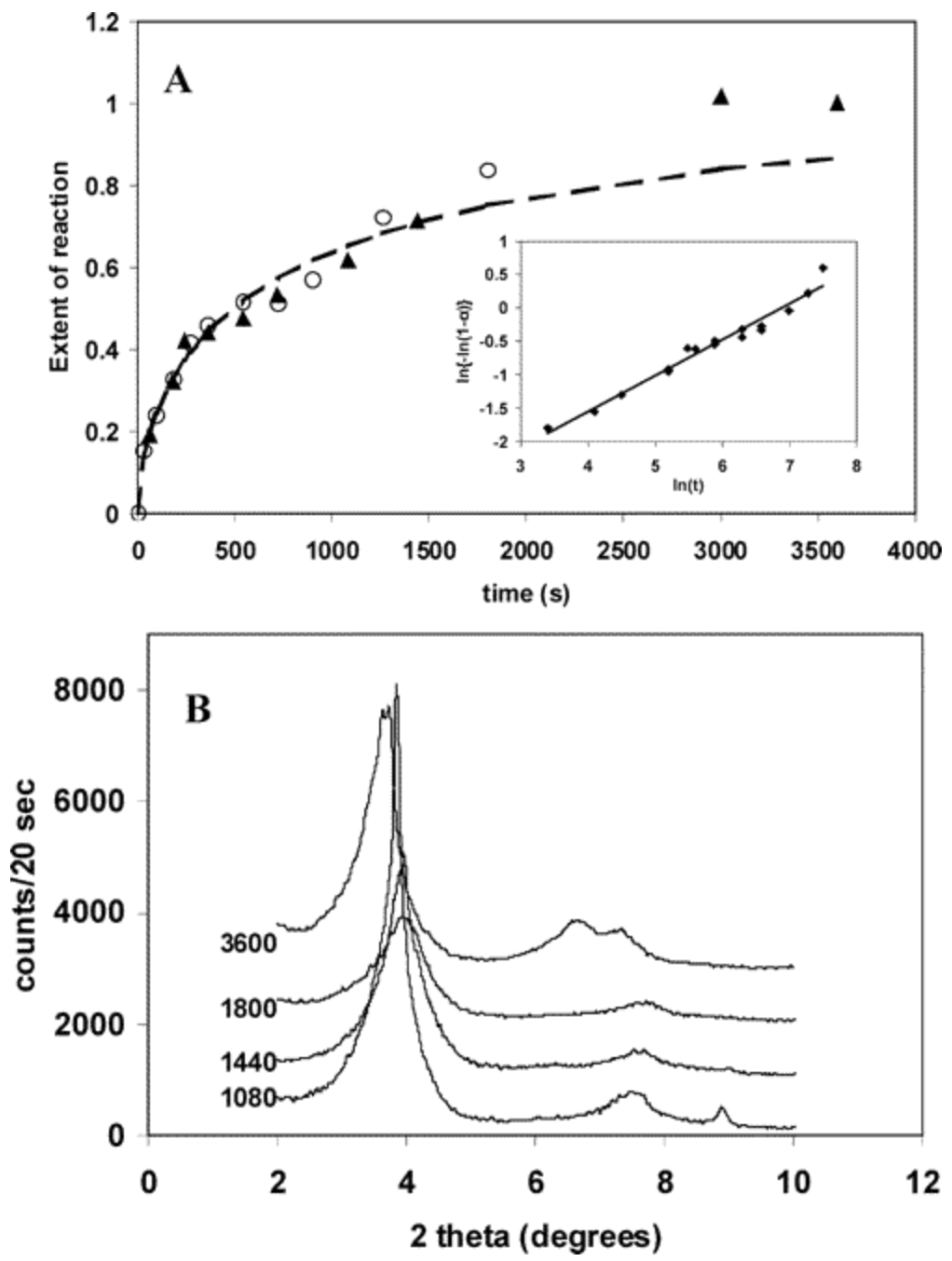

Figure 6 (A) Extent of reaction as a function of time for acetate anions released from NZA HDS exposed to octanoate solution and the corresponding Sharp-Hancock plot (inset). Data were obtained from two separately synthesized batches of NZA as indicated by $\square$ and $\circ$. Kinetic parameters derived from Sharp-Hancock analysis do not adequately model the entire time range. (B) PXRD data obtained for solid materials recovered from the reaction of NZA HDS with octanoate. Time of exposure to exchange solutions (in seconds) is indicated for each trace. Plots are offset for clarity but not otherwise scaled.

PXRD data are shown in Figure 6B for the solid-state material obtained during the time where the second stage of acetate release is observed. At $1080 \mathrm{~s}$, two broad features assigned to 001 and 002 of the product octanoate are observed at 3.8 and $7.7^{\circ}$, respectively. It is interesting that the original acetate phase 001 peak at 6.8 is no longer evident, even though only about half of the acetate has been released into solution at this point. In addition, a peak is observed at $2 \theta=9.0^{\circ}$, corresponding to a $d$ spacing of $9.8 \AA$, smaller than the $13.6-\AA$ basal

Journal of Physical Chemistry B, Vol 109, No. 17 (February 2005): pg. 8469-8475. DOI. This article is (C) American Chemical Society and permission has been granted for this version to appear in e-Publications@Marquette. American Chemical Society does not grant permission for this article to be further copied/distributed or hosted elsewhere without the express permission from American Chemical Society. 
spacing of the original HDS. The $9.0^{\circ}$ peak disappears by $1440 \mathrm{~s}$, suggesting that an intermediate phase that may be influencing the rate of acetate release. At the final time shown in Figure 6B, a new feature appears at $6.8^{\circ}$ that was not observed at the earlier times. Clearly there is a more complex set of structural transformations also taking place in the NZA reaction than would be expected for a topotactic phase transformation. An investigation of the kinetics with precursors containing other $\mathrm{Zn} / \mathrm{Ni}$ compositions will be the subject of future work, as will studies of kinetics of milled samples with varying degrees of structural disorder. We also note that the solutions remained colorless at all times during the reactions, suggesting that large-scale dissolution followed by reprecipitation of products is not a major factor.

\section{Conclusions}

Anion exchange reactions of HDSs and a related basic zinc hydroxy acetate compound are shown to exhibit a variety of kinetic behaviors that depend on the relative size of the anions as well as the structure/composition of the layered precursor. An unusual zerothorder kinetics release was observed for the replacement of acetate in ZCA by formate. The Avrami-Erofe'ev kinetic model can be used to describe rate of acetate release in the reactions of zinc copper hydroxy acetate HDS with butyrate and octanoate anions as well as the reaction of zinc hydroxy acetate with octanoate. In the case of ZCA HDS, the butyrate reaction reaches equilibrium more quickly than does the octanoate exchange $\left(k_{\text {butyrate }}=2.6 \times 10^{-3} \mathrm{~s}^{-1}\right.$ versus $k_{\text {octanoate }}=0.8$ $\times 10^{-3} \mathrm{~s}^{-1}$ ), but the octanoate reaches a final $98 \%$ conversion compared with $55 \%$ for butyrate. The butyrate reaction also produces more than one solid product structure. The reaction of ZHA with octanoate proceeds more quickly than that of ZCA with the same anion but does not go to completion, and the harvested solid phase exhibits significant disorder. The reaction of nickel zinc hydroxy acetate with octanoate does not follow Avrami-Erofe'ev kinetics over the entire reaction period. An intermediate species is postulated to be the source of this discrepancy based on PXRD data. 
NOT THE PUBLISHED VERSION; this is the author's final, peer-reviewed manuscript. The published version may be accessed by following the link in the citation at the bottom of the page.

\section{Acknowledgment}

We thank the Marquette University Committee on Research for financial assistance, D. Sem for help with the optimization of ${ }^{1} \mathrm{H}$ NMR acquisition parameters, and J. Collins for technical support of the X-ray diffractometer.

\section{References}

1Rives, V. Layered Double Hydroxides: Present and Future; Nova Science: New York, 2001 and extensive references therein.

2Fujita, W.; Awaga, K. Inorg. Chem. 1996, 35, 1915.

3.Laget, V.; Hornick, C.; Rabu, P.; Drillon, M. J. Mater. Chem. 1999, 9, 169. 4Fujita, W.; Awaga, K. J. Am. Chem. Soc. 1997, 119, 4563.

5 Morioka, H.; Tagaya, H.; Kadokawa, J.; Chiba, K. Recent. Res. Dev. Mater. Sci. 1998, 1, 137.

6Meyn, M.; Beneke, K.; Lagaly, G. Inorg. Chem. 1993, 32, 1209.

ZChoy, J.; Kwon, Y.; Song, S.; Chang, S. Bull. Korean Chem. Soc. 1997, 18, 450.

8Morioka, H.; Tagaya, H.; Karasu, M.; Kadokawa, J.; Chiba, K. Inorg. Chem. 1999, 38, 4211

9O'Brien, S.; Francis, R. J.; Fogg, A.; O'Hare, D.; Okazaki, N.; Kuroda, K. Chem. Mater. 1999, 11, 1822.

${ }^{10}$ Fogg, A. M.; O'Hare, D. Chem. Mater. 1999, 11, 1771.

11O'Hare, D.; Evans, J. S. O.; Price, S. Molecular Recognit. Inclusion 1998, 153.

12Williams, G. R.; Norquist, A. J.; O'Hare, D. Chem. Mater. 2004, 16, 975.

$\underline{13}$ Sheridan, A. K.; Anwar, J. Chem. Mater. 1996, 8, 1042.

$\underline{14}$ Fogg, A. M.; Dunn, J. S.; O'Hare, D. Chem. Mater. 1998, 10, 356.

$\underline{15}$ Evans, J. S. O.; Price, S. J.; Wong, H.; O'Hare, D. J. Am. Chem. Soc. 1998 , $120,10837$.

16Khan, A. I.; Lei, L.; Norquist, A. J.; O'Hare, D. Chem. Commun. 2001, 2342.

17Ambrogi, V.; Fardella, G.; Grandolini, G.; Perioli, L. Int. J. Pharm. 2001, 220, 23.

$\underline{18}$ Cheary, R. W.; Coelho, A. A. Programs XFIT and FOURYA, deposited in the CCP14 Powder Diffraction Library, Engineering and Physical Sciences Reasearch Council, Daresbury Laboratory, Warrington, England (http://www.ccp14.ac.uk/tutorials/xfit- 95/xfit.htm), 1996.

$\underline{19}$ Rojas, R.; Barriga, C.; Ulibarri, M. A.; Malet, P.; Rives, V. J. Mater. Chem. 2002, 12, 1071

${ }^{20}$ Rajamathi, M.; Nataraja, G. D.; Ananthamurthy, S.; Kamath, P. V. J. Mater. Chem. 2000, 10, 2754.

Journal of Physical Chemistry B, Vol 109, No. 17 (February 2005): pg. 8469-8475. DOI. This article is (C) American Chemical Society and permission has been granted for this version to appear in e-Publications@Marquette. American Chemical Society does not grant permission for this article to be further copied/distributed or hosted elsewhere without the express permission from American Chemical Society. 
NOT THE PUBLISHED VERSION; this is the author's final, peer-reviewed manuscript. The published version may be accessed by following the link in the citation at the bottom of the page.

21]Jenkins, R.; Synder, R. L. Introduction to X-ray Powder Diffractometry; Wiley: New York, 1996.

22Nakamoto, K. Infrared and Raman Spectra of Inorganic and Coordination Compounds, 4th ed.; Wiley: New York, 1986; p 232.

23. Deacon, G. B.; Phillips, R. J. Coord. Chem. Rev. 1980, 33, 227.

24Poul, L.; Jouini, N.; Fievet, F. Chem. Mater. 2000, 12, 3123.

$\underline{25}$ Masciocchi, N.; Corradi, E. ; Sironi, A.; Moretti, G.; Minelli, G. ; Porta, P. J. Solid State Chem. 1997, 131, 252.

26 Laugier J.; Bochu, B. LMGP-Suite Suite of Programs for the Interpretation of $X$-ray Experiments; ENSP/Laboratoire des Matériaux et du Génie Physique, BP 46. 38042: Saint Martin d'Hères, France

(http://www.inpg.fr/LMGP and http://www.ccp14.ac.uk/tutorial/lmgp/).

27Cao, G.; Mallouk, T. E. Inorg. Chem. 1991, 30, 1434.

28Avrami, M. J. Chem. Phys. 1940, 8, 212.

29Avrami, M. J. Chem. Phys. 1941, 9, 177.

${ }^{30}$ Brown, W. E.; Dollimore, D.; Galwey, A. K. Reactions in the Solid State. In Comprehensive Chemical Kinetics; Bamford, C. H., Tipper, C. F. H., Eds.; Elsevier: Amsterdam, 1980; Vol. 22, pp 40-113.

31Walton, R. I.; O'Hare, D. J. Phys. Chem. B 2001, 105, 91

32Dutta, P. K.; Puri, M. J. Phys. Chem. 1989, 93, 376.

33$A$ Aberti, G.; Costantino, U. Layered Solids and Their Intercalation Chemistry. In Comprehensive Supramolecular Chemistry: Two- and ThreeDimensional Networks; Alberti, G., Bein, T., Eds.; Elsevier Science Ltd: Great Britain, 1996; Vol. 7, p 11.

\section{Supporting Information Available}




\section{Supporting Materials}
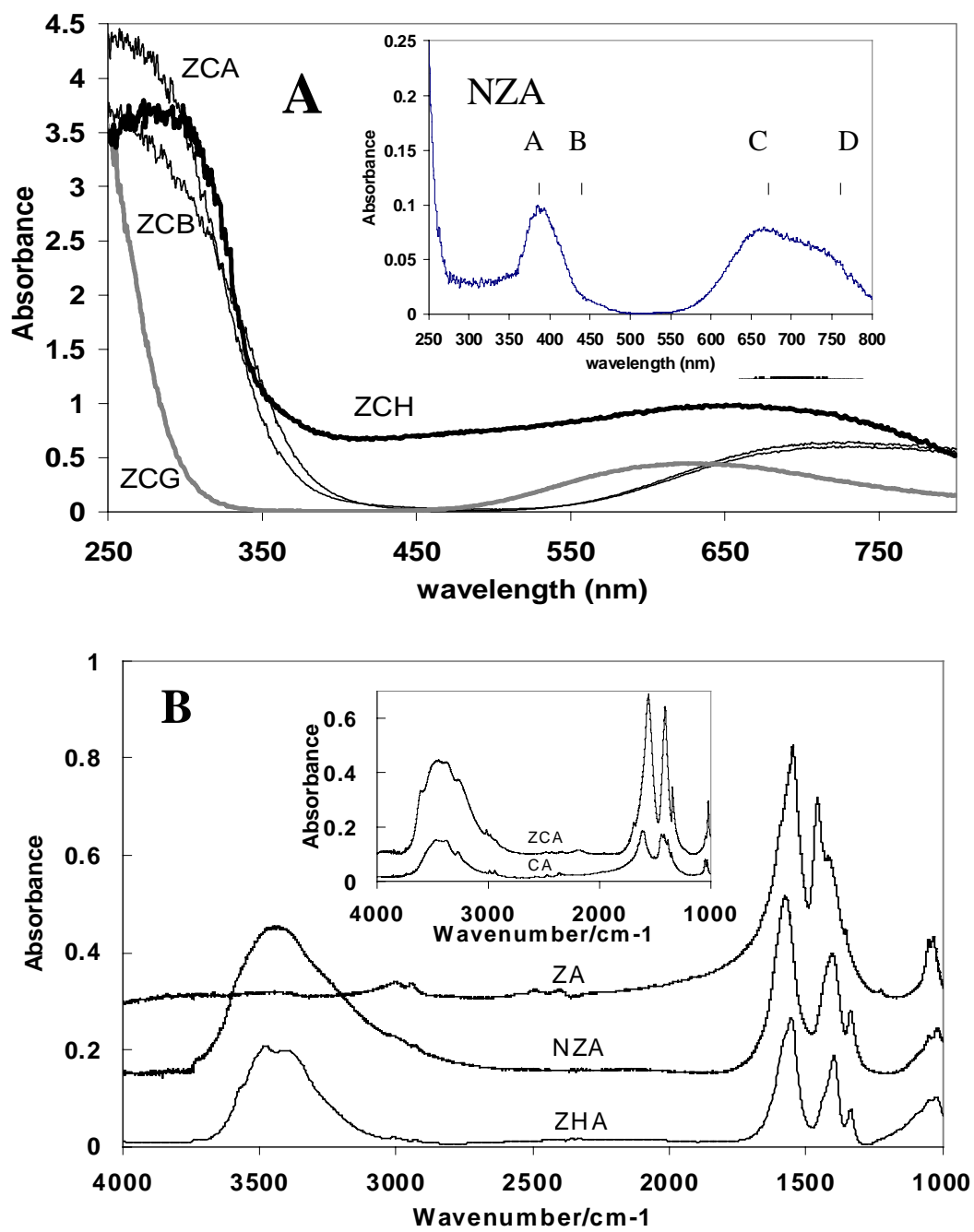

Figure S1. (A) Diffuse reflectance spectra of ZCA, zinc copper butyrate (ZCB), zinc copper cinnamate $(\mathrm{ZCH})$ and zinc copper glutamate $(\mathrm{ZCG}) \mathrm{HDS}$. Inset shows DRS of NZA HDS. Positions of bands assigned in reference 20 are marked $A \quad\left(v_{3}:{ }^{3} \mathrm{~T}_{1 \mathrm{~g}}(\mathrm{P}) \leftarrow^{3} \mathrm{~A}_{2 \mathrm{~g}}(\mathrm{~F})\right)$, $\mathrm{B}$ $\left({ }^{1} \mathrm{~T}_{1 \mathrm{~g}}(\mathrm{G}) \longleftarrow{ }^{3} \mathrm{~A}_{2 \mathrm{~g}}(\mathrm{~F})\right)$, C $\left({ }^{1} \mathrm{E}_{\mathrm{g}}(\mathrm{G}){ }^{3} \mathrm{~A}_{2 \mathrm{~g}}(\mathrm{~F})\right)$, and $\mathrm{D}\left(\mathrm{v}_{2}:{ }^{3} \mathrm{~T}_{1 \mathrm{~g}}(\mathrm{~F}) \leftarrow{ }^{3} \mathrm{~A}_{2 \mathrm{~g}}(\mathrm{~F})\right)$. (B) FTIR spectra of layered compounds and reference metal acetate precursors. 
Table S1. Carboxylate vibrational frequencies

\begin{tabular}{lllccc}
\hline \hline Species & $v_{\text {as }}\left(\mathrm{cm}^{-1}\right)$ & $v_{\mathrm{s}}\left(\mathrm{cm}^{-1}\right)$ & $\Delta v_{\text {as-s }}\left(\mathrm{cm}^{-1}\right)$ & $\begin{array}{l}\Delta v_{\text {as-s }}\left(\mathrm{cm}^{-1}\right) \\
\text { Literature }\end{array}$ & $\begin{array}{c}\text { Lit. } \\
\text { Ref. }\end{array}$ \\
\hline Zn acetate & 1548 & 1457 & 91 & 94 & A \\
Cu acetate & 1605 & 1420 & 185 & 185 & B \\
ZHA & 1549 & 1394 & 155 & 172 & C* \\
ZCA & 1563 & 1410 & 153 & - & - \\
NZA & 1577 & 1408 & 169 & 166 & D \\
\hline
\end{tabular}

*Synthesized via hydrolysis in polyol medium.

References:
A. Deacon, G. B.; Phillips, R. J. Coord. Chem. Rev. 1980, 33, 227.
B. Nakamoto, K.; Fujita, J.; Tanaka, S.; Kobayashi, M. J. Am. Chem. Soc. 1957, 79,4904
C. Poul, L.; Jouini, N.; Fievet, F. Chem. Mater. 2000, 12(10), 3123.
D. Rojas, R.; Barriga, C.; Ulibarri, M. A.; Malet, P.; Rives, V. J. Mater. Chem. 2002, 12,1071 .

